\title{
Chilaiditi's sign
}

\author{
Kaushik Saha*
}

NRS Medical College and Hospital, Kolkata, West Bengal, India

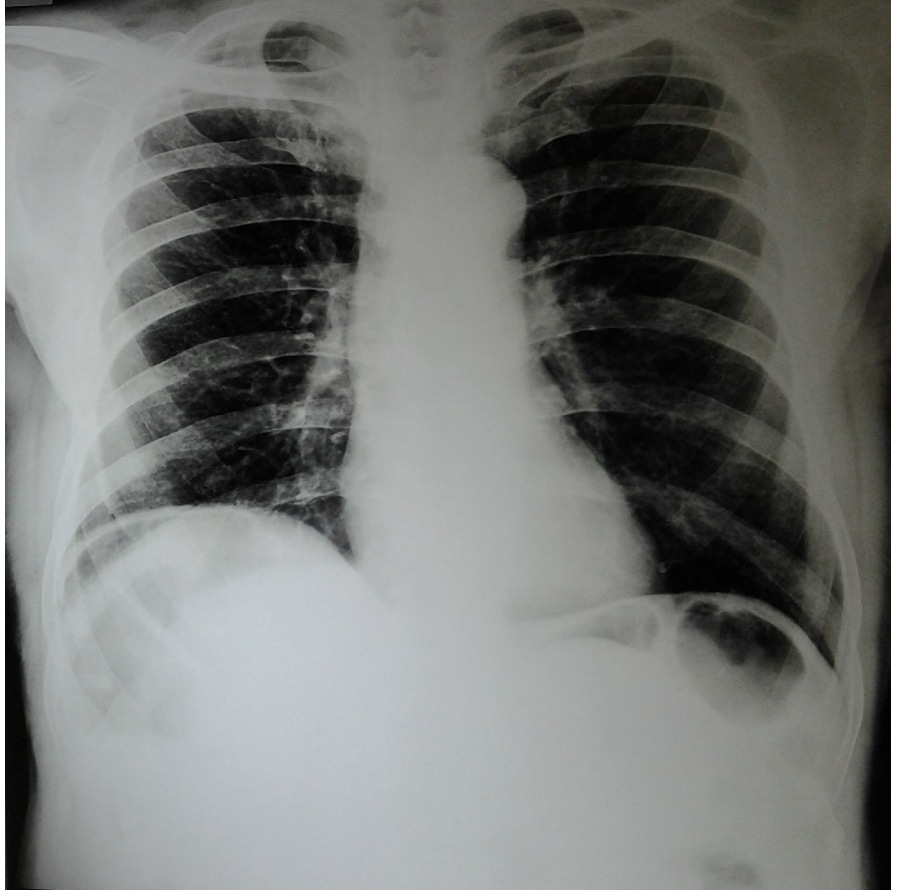

Figure 1:

Chest X-ray PA view

\section{Accepted on}

November $20^{\text {th }}, 2012$

\section{DOI Name}

10.3126/jaim.v2i1.7632

\section{Keywords}

COPD, chilaiditi's sign, chest $x$-ray.

\section{Citation}

Kaushik Saha. Chilaiditi's sign. Journal of Advances in Internal Medicine 2013;02(01):16.

\section{* Corresponding author}

Department of Pulmonary Medicine,

NRS Medical College and Hospital, 138, AJC Bose Road, Kolkata, West Bengal, India.

Email address - doctorkaushiksaha@gmail.com

\section{CASE}

A 70-year-old male patient, who was a known case of chronic obstructive pulmonary disease (COPD) presented with increase in severity of cough and shortness of breath for 7 days. His physical findings were consistent with the diagnosis of COPD. Investigations like blood for complete hemogram, serum urea \& creatinine, electrocardiography and ultrasonography of abdomen were unremarkable. His chest X-ray PA view is shown in figure 1. What is the unexpected finding in chest X-ray \& what is its significance?

\section{ANSWER}

Chest X-ray PA view shows colonic gas under right dome of diaphragm suggestive of chilaiditi's sign. Free air under diaphragm produces a continuous crescent shaped translucency under the diaphragm. This radiograph revealed haustral pattern of subdiaphragmatic translucency. It is due to interposition of a loop of transverse colon between liver and diaphragm visible on chest X-ray or abdominal X-ray. This is known as Chilaiditi's sign. ${ }^{1}$

The incidence on chest X-ray or abdominal X-rays are around $0.1 \%$ but in older adults it can be found up to $1 \% .{ }^{1}$ Most of the patients are usually asymptomatic. Chiladiti's syndrome means presence of complications along with chiladiti's sign. ${ }^{2}$ This syndrome is usually due to absence or laxity of the falciform ligament or suspending ligament of the transverse colon. It may occur in patients with chronic lung disease, chronic liver disease such as cirrhosis, ascites. This condition may be mistaken as acute abdomen due to peptic perforation and operative interference may be sought. ${ }^{3}$ This sign has to be kept in mind before any surgical interference.

\section{REFERENCES}

1. Sanyal K, Sabanathan K. Air below the right diaphragm: Chilaiditi sign. Emerg Med J 2008;25:300.

2. Saber AA, Boros MJ. Chilaiditi's syndrome: what should every surgeon know? Am J Surg 2005;71:261-3.

3. Glatter RD, April RS, Miskovitz P, et al. Severe recurrent abdominal pain: an anatomical variant of Chilaiditi's syndrome. MedGenMed 2007;9:67. 